\title{
Universality of the companion mass-ratio distribution (Research Note)
}

\author{
M. Reggiani and M. R. Meyer \\ Institute of Astronomy, ETH Zurich, 8093 Zurich, Switzerland \\ e-mail: reggiani@phys.ethz.ch \\ Received 3 April 2013 / Accepted 12 April 2013

\begin{abstract}
Aims. We present new results regarding the companion mass-ratio distribution (CMRD) of stars, as a follow-up of our previous work. Methods. We used a maximum-likelihood-estimation method to re-derive the field CMRD power law avoiding dependence on the arbitrary binning. We also considered two new surveys of multiples in the field for solar-type stars and M dwarfs to test the universality of the CMRD.

Results. We found no significant differences in the CMRD for M dwarfs and solar-type stars compared with previous results over the common mass ratio and separation range. The new best-fit power law of the CMRD in the field, combining two previous sets of data, is $\mathrm{d} N / \mathrm{d} q \propto q^{\beta}$, with $\beta=0.25 \pm 0.29$.
\end{abstract}

Key words. binaries: general - stars: formation - stars: low-mass - stars: solar-type

\section{Introduction}

A large portion of stars, both in the field (Raghavan et al. 2010; Janson et al. 2012) and in star-forming regions (Patience et al. 2002), are formed in multiple systems. Therefore understanding multiple star formation is necessary to investigate star formation in general (Goodwin et al. 2007; Duchêne \& Kraus 2013). Because binary properties reflect the main characteristics of binary formation, they may help us determining the most common mechanisms for the formation of multiple stars. In a binary system of stars with masses $M_{1}$ and $M_{2}\left(M_{1}>M_{2}\right)$, the mass-ratio is conventionally defined as $q=M_{2} / M_{1}$. Similar to the initial mass function (IMF) for single objects, the companion massratio distribution (CMRD) is the distribution of $q$ values as a function of primary mass. Tidal capture models predict that for each primary star the mass of the secondary is chosen randomly from the single-star mass function, and the CMRD reflects the IMF (McDonald \& Clarke 1993; Kroupa et al. 2003). In fragmentation scenarios subsequent continued accretion onto both objects from a common reservoir tends to equalize the masses, resulting in a $q$ distribution peaked toward unity (Bate 2000). Capture is unlikely to be the most relevant binary formation mechanism, but it may still occur during the dissolution phase of star clusters, causing differences in the shape of the CMRD as a function of orbital separation (Moeckel \& Bate 2010; Moeckel \& Clarke 2011). Motivated by the fact that every theoretical model predicts a different shape of the mass-ratio distribution and of dependency of the CMRD on the primary mass, we used MonteCarlo simulations to compare the CMRD for different samples and to study the relationship between the IMF and the CMRD (Reggiani \& Meyer 2011, hereafter RM11). This research note represents a follow-up to RM11, in which we reanalyze the "universal" CMRD by adopting a different statistical approach
(Sect. 2) and some new results on the CMRD on the basis of recent datasets (Sect. 3).

\section{Universal companion mass-ratio distribution}

The CMRD appears to be universal over a wide range of $q$ values and primary masses (e.g. Metchev \& Hillenbrand 2009). According to RM11, the CMRD follows a single-slope power law $\mathrm{d} N / \mathrm{d} q \propto q^{\beta}$ over the separation range 1-2400 AU and primary mass range $0.25-6.5 M_{\odot}$, and there is no evidence for variation of the CMRD with orbital separation.

In previous work we combined samples of M dwarfs (Fischer \& Marcy 1992) and G stars (Metchev \& Hillenbrand 2009) in the field and intermediate mass stars in ScoOB2 (Kouwenhoven et al. 2005) adopting a $\chi^{2}$ fit of the combined binned distribution to derive the power law slope, obtaining $\beta=-0.50 \pm 0.29$ (Reggiani \& Meyer 2011). The choice of the statistical method was motivated by the need of comparing our results with previous studies of the CMRD (e.g. Kouwenhoven et al. 2005; Metchev \& Hillenbrand 2009). However, the $\chi^{2}$ fit of a binned distribution can lead to a biased estimate, in particular for small samples. A more robust analysis is instead achieved through a maximum-likelihood estimation method (Feigelson \& Babu 2011). This approach gives a new best-fit power law $\mathrm{d} N / \mathrm{d} q \propto$ $q^{\beta}$, with $\beta=-0.18 \pm 0.33$ to the data described in RM11. Although the two values are consistent with each other within the errors, the new estimate is flatter than previously thought.

\section{Updates to the CMRD in the field}

Recently, two new studies of the CMRD for solar-type (Raghavan et al. 2010) and M-dwarf primaries (Janson et al. 2012) in the field have been carried out. Since they represent the 
most complete samples to date for sun-like stars and M dwarfs, respectively, we applied the same statistical analysis as was presented in RM11 to follow up this preliminary work.

In the first study (Raghavan et al. 2010), roughly 200 binaries with primary masses between $0.5-3 M_{\odot}$ were considered to determine the CMRD over a wide range of separations $\left(10^{-1}-10^{5} \mathrm{AU}\right)$ and mass ratios $(0.02-1)$. The new CMRD appears to be more peaked toward unity than previously observed and the period distribution is unimodal and roughly log-normal with a peak at around 50 AU. Following the methodology described in RM11, we used a KS test to compare the newly observed CMRD with the CMRD by Metchev \& Hillenbrand (2009), over the common range of mass ratios (0.02-1) and separations (28-1590 AU). The KS test returns a probability of $\sim 30 \%$, therefore we cannot reject the hypothesis that the data were drawn from the same parent population. However, when we compare the two samples over the common range of mass ratios, irrespective of separation, the probability is only $\sim 1 \%$, pointing toward a change of the CMRD with orbital radius, because that of Raghavan et al. (2010) covers a wider range than that of Metchev \& Hillenbrand (2009). We therefore tested the possibility of a variation of the CMRD with angular separation. To do this, we considered break points in the angular separation distribution between $10^{-1}$ and $10^{5} \mathrm{AU}$ and used a KS test for each of them to determine the probability that the CMRD inside the break point is consistent with the CMRD outside. Because we found probabilities greater than $1 \%$ for any possible choice of break point, we conclude that we have no strong evidence for a dependence of the CMRD on angular separation. Moreover, because we do not expect to see random pairing from cluster dissolution models inside $10^{4} \mathrm{AU}$ (Kouwenhoven et al. 2010) and these widest binaries are relatively rare, we need larger samples in the future to test these models.

The second study (Janson et al. 2012) consists of 85 systems with primary masses between $0.15-0.5 M_{\odot}$, separations in the range 3-227 $\mathrm{AU}$ and mass ratios between 0.1 and 1. For M dwarfs, the CMRD appears to be flat and the period distribution is narrower and peaks at lower values than for solar type primaries, indicating a continuous transition from higher- to lower-mass stars (Burgasser et al. 2007). In this case as well, we tested the newly observed CMRD with the CMRD from Fischer $\&$ Marcy (1992) over the common range of mass ratios and separations. With a probability of $\sim 56 \%$ the KS test does not allow us to reject the hypothesis that the newer data were drawn from the same parent sample. Finally, we used the same procedure as we adopted for sun-like stars, but in the range 3-227 AU, to explore the dependence of the observed CMRD on separation. We saw no evidence of this dependence for this sample either.

Moreover, we compared the CMRD for solar-type primaries from Raghavan et al. (2010) with the new sample of M-dwarf primary binaries (Janson et al. 2012). The KS test returned a probability of $30 \%$ that the two distributions are consistent with each other (Fig. 1). Motivated by this result and because the CMRD is independent of angular separation, we combined the two CMRDs over the common range of mass ratios. We again used a maximum-likelihood method to fit the distribution and found a power law $\mathrm{d} N / \mathrm{d} q \propto q^{\beta}$, with $\beta=0.25 \pm 0.29$. While this slope $\beta$ is formally consistent with the one derived in Sect. 2 (within the errors), the change in sign is significant. It is also worth mentioning that this fit is consistent with the mass-ratio distribution with power-law exponent $\beta=-0.10 \pm 0.58$ presented in a recent study of O-type spectroscopic binaries (Sana et al. 2012), whereas the observed CMRD for brown dwarfs

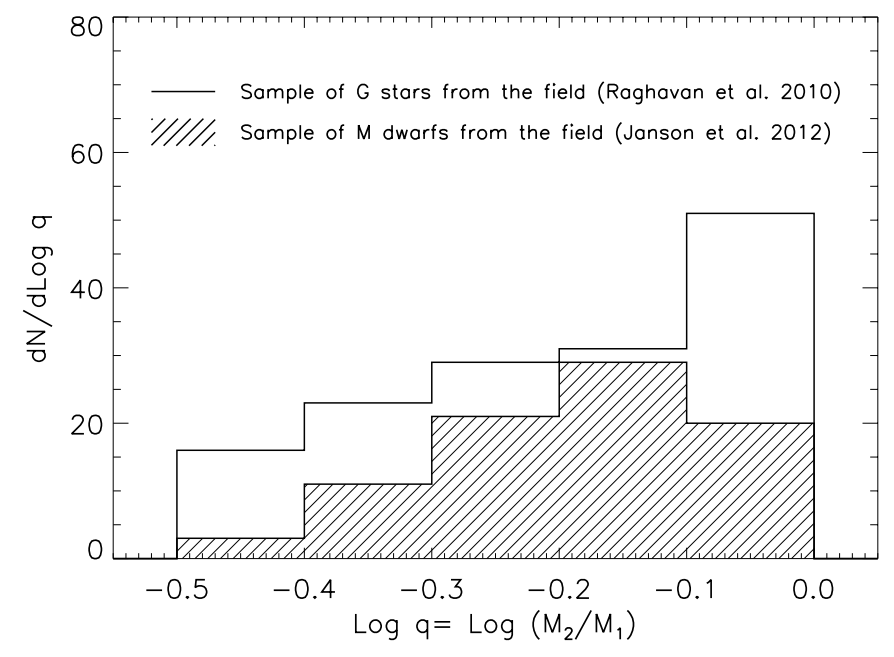

Fig. 1. Comparison between the observed CMRDs for solar-type primaries and M-dwarf primaries in the field. The open histogram represents the CMRD from Raghavan et al. (2010), whereas the hashed histogram represents the distribution from Janson et al. (2012). The KS test returns a probability of $30 \%$ that the two distributions are drawn from the same parent sample.

$(\beta \sim 1.5)$ points toward a different formation mechanisms for these objects (Goodwin 2013).

\section{Summary}

In this research note we have presented some updates to the study of RM11. First, we adopted a maximum-likelihood estimation method to re-derive the field CMRD power law, based on the combination of samples (Fischer \& Marcy 1992; Metchev \& Hillenbrand 2009; Kouwenhoven et al. 2005) described in RM11, to show how the dependence on the bin size can bias the result.

Secondly, we analyzed recent binarity studies from the field (Raghavan et al. 2010; Janson et al. 2012) adopting the same methodology as in RM11. The new results from Raghavan et al. (2010) appear to be consistent with Metchev \& Hillenbrand (2009) over the common range of mass ratios and angular separations. The recent updates on the M-dwarf CMRD (Janson et al. 2012) are also consistent with past results. The KS test does not allow us to reject the hypothesis that the CMRDs from Raghavan et al. (2010) and Janson et al. (2012) are drawn from the same parent sample. In both studies we uncovered no evidence for a dependence of the CMRD on separation. Therefore we combined the two distributions and obtained a new maximum-likelihood fit to the field CMRD $\mathrm{d} N / \mathrm{d} q \propto q^{\beta}$, with $\beta=0.25 \pm 0.29$.

Since the CMRD appears to be independent of separation and dynamical evolution (see also Parker \& Reggiani 2013), it represents a measurable parameter of binary stars to focus on when investigating binary formation mechanisms. However, we need larger samples to look for subtle variations of the CMRD with separation. In the future we aim to study the CMRD in other star-forming regions (e.g. the ONC) and test its dependence on separation for wide systems.

Acknowledgements. We thank the referee, Simon Goodwin, for his review. We are also grateful to Carolina Bergfors, Stanimir Metchev, Deepak Raghavan, Eric Feigelson, and Richard Parker for the insightful discussions and sharing their data electronically. 
M. Reggiani and M. R. Meyer: Universality of the CMRD $(R N)$

\section{References}

Bate, M. R. 2000, MNRAS, 314, 33

Burgasser, A. J., Reid, I. N., Siegler, N., et al. 2007, Protostars and Planets V, 427

Duchêne, G., \& Kraus, A. 2013, ARA\&A, in press [arXiv: 1303.3028]

Feigelson, E. D., \& Babu, G. J. 2011, Modern Statistical Methods for Astronomy with R Applications, Cambridge Univ. Press

Fischer, D. A., \& Marcy, G. W. 1992, ApJ, 396, 178

Goodwin, S. P. 2013, MNRAS, 430, L6

Goodwin, S. P., Kroupa, P., Goodman, A., \& Burkert, A. 2007, Protostars and Planets V, 133

Kouwenhoven, M. B. N., Brown, A. G. A., Zinnecker, H., Kaper, L., \& Portegies Zwart, S. F. 2005, A\&A, 430, 137
Kouwenhoven, M. B. N., Goodwin, S. P., Parker, R. J., et al. 2010, MNRAS, 404, 1835

Kroupa, P., Bouvier, J., Duchêne, G., \& Moraux, E. 2003, MNRAS, 346, 354

Janson, M., Hormuth, F., Bergfors, C., et al. 2012, ApJ, 754, 44

McDonald, J. M., \& Clarke, C. J. 1993, MNRAS, 262, 800

Metchev, S. A., \& Hillenbrand, L. A. 2009, ApJS, 181, 62

Moeckel, N., \& Bate, M. R. 2010, MNRAS, 404, 721

Moeckel, N., \& Clarke, C. J. 2011, MNRAS, 415, 1179

Parker, R. J., \& Reggiani, M. M. 2013, MNRAS, accepted [arXiv: 1304.3123]

Patience, J., Ghez, A. M., Reid, I. N., \& Matthews, K. 2002, AJ, 123, 1570

Raghavan, D., McAlister, H. A., Henry, T. J., et al. 2010, ApJS, 190, 1

Reggiani, M. M., \& Meyer, M. R. 2011, ApJ, 738, 60

Sana, H., de Mink, S. E., de Koter, A., et al. 2012, Science, 337, 444 